\title{
TRITERPENOS Y ESTEROLES DE Salvia leucantha (LAMIACEAE) Y EVALUACIÓN DE SU CAPACIDAD ANTIOXIDANTE
}

Fecha de recepción: 10 de abril de 2014 • Fecha de aceptación: 30 de mayo de 2014

TRITERPENES AND STEROLS OF Salvia leucantha (LAMIACEAE) AND EVALUATION OF THEIR ANTIOXIDANT CAPACITY

Willy Fernando Cely Veloza ${ }^{1,2}$, Javier A. Matulevich', William F. Castrillón ${ }^{1}$

\section{RESUMEN}

Con el fin de contribuir en el conocimiento de la flora Colombiana, particularmente aquella presente en la región del Guavio, se eligió la especie Salvia leucantha para realizar un estudio fitoquímico preliminar de su composición química y bioactividad a nivel antioxidante. Para ello se realizó el análisis fitoquímico preliminar de hojas y se evaluó la presencia de los principales grupos de metabolitos secundarios, cuyas estructuras fueron analizadas tentativamente por CG-EM y respectiva comparación con las bases de datos NIST-08, adicionalmente se evaluó la actividad antioxidante de extractos y fracciones por DPPH. Se detectó la presencia de triterpenos pentaciclicos en gran concentración, lo cual era de esperarse al tratarse de una especie del género Salvia, ampliamente conocida por su contenido de terpenos y amplia gama de aceites esenciales. Estos compuestos son ampliamente utilizados en la industria alimenticia, cosmética y farmacéutica por sus propiedades físico-químicas y farmacológicas, pero no reportan porcentajes de inhibición altos para poderlos catalogar como antioxidantes fuertes. Los resultados de este trabajo son importantes como primera aproximación a la ampliación del conocimiento químico y a la posible utilidad de las especies presentes en esta zona de Colombia con características agronómicas óptimas para el establecimiento de cultivos, que permitan una utilización racional de los recursos naturales de nuestro país, como fuente de desarrollo regional y nacional.

Palabras clave: Triterpenos, Esteroles, CG-EM, Capacidad antioxidante.

1. Grupo de investigación en Productos Naturales, Universidad Distrital Francisco José de Caldas, Bogotá, Colombia.

2. Autor Corresponsal: mikerinos66@hotmail.com 


\begin{abstract}
In order to improve our understanding of the Colombian flora, particularly those present in the region of Guavio, the specie Salvia leucantha was selected for further preliminary phytochemical studies, chemical composition studies and antioxidant bioactivity. Preliminary phytochemical analysis of leafs was carried out to establish the main groups of secondary metabolites presents, those were analyzed by GC-MS comparing the obtained spectra with the NIST-08 database, in addition antioxidant activity was determined using DPPH method. High amounts of pentacyclic triterpenes was determined according to expectations of the genere Salvia, rich in essential oils and this kind of compounds. This family of compounds is widely used in food, cosmetics and pharmaceutical industry due to its physicochemical and pharmacological properties; unfortunately they do not report high inhibition percentages to be classified as strong antioxidants. The present work shows the results as a first approximation to expansion of chemical knowledge of various Colombian species.
\end{abstract}

Keywords: Triterpenes, steroles, GC-MS, Antioxidant capacity.

\title{
INTRODUCCIÓN
}

La familia Lamiaceae tiene importancia económica mundial, ya que básicamente en todos los continentes y culturas el hombre ha utilizado numerosas especies de labiadas como medicina, condimento o alimento. También han sido usadas en numerosos casos como plantas ornamentales muy apreciadas por su aroma y por sus flores. Sólo por citar algunas de las labiadas más comúnmente usadas en nuestro medio tenemos plantas como la albahaca (Ocimum), alegrías (Scutellaria), marrubio (Marrubium), hierbabuena, menta y poleo clásico (Mentha), orégano clásico y mejorana (Origanum), orégano orejón (Plectranthus), orégano andino (Mintostachys), ajedrea y poleo americano (Satureja), Salvia (Salvia y Lepechinia), mastranto (especies de Hyptis y Salvia), tomillo (Satureja, Thymus), toronjil (Melissa) y romero (Rosmarinus). Por otra parte, algunas labiadas son tradicionalmente utilizadas en los ritos mágicos religiosos de algunas comunidades, como es el caso de varias especies de los géneros Scutellaria y Ocimun, principalmente en las comunidades afrocolombianas e indígenas del litoral pacífico. Son también frecuentes en nuestros parques y jardines algunas especies ornamentales introducidas, de los géneros Ajuga, Leonotis, Mentha, Ocimum, Plectranthus, Salvia, Scutellaria, Stachys y Teucrium, y algunas especies nativas del género Salvia (Fernandez-Alonso, 2006).

Son numerosas las actividades biológicas que se han reportado en la familia de las labiadas dentro de las cuales vale la pena mencionar la actividad antimicrobiana, anti fúngica y actividad antioxidante (Esmeralda et al., 2010). Esta última es quizá una de las más importantes ya que durante los últimos años los campos de aplicación de los antioxidantes son muy variados, además de terapias farmacológicas, también tienen interés como suplementos nutricionales, ingredientes cosméticos para prevenir 
el envejecimiento cutáneo, alimentos funcionales y como conservantes alimentarios. La industria agroalimentaria se enfrenta continuamente a problemas de estabilidad de alimentos derivados de la oxidación de los mismos. El uso de antioxidantes sintéticos está muy generalizado pero existe controversia debido a que algunos presentan problemas de toxicidad por ello los antioxidantes naturales están constituyendo en la actualidad una alternativa y por esta razón que se han adelantado numerosos estudios en diferentes familias vegetales a fin de analizar sus propiedades antioxidantes (Esmeralda et al., 2010).

\section{MATERIALES Y MÉTODOS}

\section{Material Vegetal}

El material vegetal se recolectó en el municipio de Chipaque en Cundinamarca a una altura de 2668 msnm cuyas coordenadas son: Latitud 04 56' 10.58" $\mathrm{N}$, Longitud $73^{\circ} 50^{\prime} 5.97^{\prime \prime} \mathrm{O}$. El municipio presenta un clima templado, óptimo para la adaptación de la especie en estudio y alcanza temperaturas que oscilan los 17 y $20^{\circ} \mathrm{C}$. Los ejemplares se recolectaron de acuerdo a los parámetros indicados en el Jardín Botánico José Celestino Mutis. La clasificación taxonómica de la especie vegetal la realizó el biólogo Gustavo
Morales, quien adicionó la especie en estudio al herbario de colecciones del Jardín Botánico de Bogotá bajo el código: WFCV-001. El nombre de la especie clasificada fue Salvia leucantha, especie perteneciente al género Salvia y a la familia de las labiadas.

\section{Preparación de Extractos Crudos}

$507 \mathrm{~g}$ de material vegetal (hojas) seco y molido fueron sometidos a una extracción por maceración en frio con éter de petróleo durante un mes reconstituyendo el solvente cada 72 horas, los extractos fueron concentrados a presión reducida en un rotaevaporador obteniendo el extracto éter de petróleo (E. EdP) (12,7011g). El material vegetal utilizado durante la maceración con EdP se dejó secar a temperatura ambiente y se sometió a una segunda extracción con etanol aplicando el mismo procedimiento del extracto anterior obteniéndose el extracto etanólico (E. EtOH) (38.098 g).

\section{Ensayo Fitoquímico Preliminar del E. EtOH.}

Se realizó un análisis Fitoquímico cualitativo para alcaloides, taninos, compuestos fenólicos, triterpenos y esteroides, flavonoides, saponinas, cumarinas y glucósidos cardiotónicos, siguiendo los parámetros reportados por Ugochukwu et al., 2013.

\section{El uso de antioxidantes sintéticos está muy generalizado pero existe controversia debido a que algunos presentan problemas de toxicidad por ello los antioxidantes naturales están constituyendo en la actualidad una alternativa y por esta razón se han adelantado numerosos estudios en diferentes familias vegetales a fin de analizar sus propiedades antioxidantes.}




\section{Fraccionamiento y purificación}

Los extractos se fraccionaron por cromatografía en columna (CC) repetitiva utilizando como fases móviles diferentes mezclas de disolventes que en ensayos previos por cromatografía en capa fina (CCD), permitieran una buena separación y selectividad, lo cual dependió de la naturaleza del extracto/fracción.

\section{Cromatografía de gases acoplada a espectrometría de masas (CG-EM)}

El análisis se llevó a cabo en un Cromatógrafo de gases SHIMADZU QP-2010 PLUS, equipado con un puerto de inyección split/splitess $\left(250^{\circ} \mathrm{C}\right.$, relación de split10:1), y un inyector automático Shimadzu AOC20i. Se utilizó una columna capilar SHRXI-5MS (SHIMADZU) de $30 \mathrm{~m} \times 0,25 \mathrm{~mm}$ (ID) $\times 0,25 \mu \mathrm{m}$ (df), con fase estacionaria $5 \%$ fenilo $95 \%$ dimetilpolisiloxano. La programación de temperatura del horno fue de $50{ }^{\circ} \mathrm{C}(2 \mathrm{~min})$ a $15^{\circ} \mathrm{C} / \mathrm{min}$, hasta $200^{\circ} \mathrm{C}(2 \mathrm{~min})$, finalmente, la temperatura aumentó a $10^{\circ} \mathrm{C} / \mathrm{min}$ hasta alcanzar $300^{\circ} \mathrm{C}$ (2 min). Los espectros de masas se obtuvieron por ionización electrónica (EI) con una energía de 70 eV. Las temperaturas de la cámara de ionización y de la línea de transferencia fueron de 230 y $275^{\circ} \mathrm{C}$, respectivamente. El gas de arrastre utilizado fue helio (Aga Fano, S.A, grado 5,0), con flujo constante de $1,2 \mathrm{~mL} / \mathrm{min}$

\section{Capacidad antioxidante (captación de radicales DPPH)}

Se prepararon soluciones de los extractos secos y fracciones a una concentración $25 \mathrm{mg} / \mathrm{mL}$. Posteriormente se preparó una solución de DPPH de trabajó disuelta en metanol a una concentración $10 \mathrm{mM}$. Posterior mente se tomaron alícuotas de extractos y fracciones correspondientes a 50, 100,
150, 200 y $250 \mu \mathrm{L}$ y $1750 \mu \mathrm{L}$ de DPPH (Brand-Williams et al., 1995). Las medidas se realizaron por triplicado en un espectrofotómetro a una longitud de onda de $517 \mathrm{~nm}$ durante una hora y el $\mathrm{IC}_{50}$ se determinó con ayuda del programa Graph phad prism. El patrón de comparación para la construcción de la curva de calibración de referencia fue ácido ascórbico.

\section{RESULTADOS Y DISCUSIÓN}

\section{Análisis Fitoquímico Preliminar}

Los resultados obtenidos en la análisis Fitoquímico preliminar fueron clasificados según el protocolo propuesto por Ugochukwu et al., 2013, en donde (-) significa NO detectado y (+) significa detectado. Dichos resultados se muestran en la tabla numero 1 :

Tabla 1. Análisis Fitoquimico Preliminar de Salvia leucantha hojas

\begin{tabular}{cc}
\hline Grupo de Metabolitos & Resultadoa \\
\hline Flavonoides & + \\
\hline Fenoles & + \\
\hline Triterpenos y Esterioides & + \\
\hline alcaloides & - \\
\hline Cumarinas & - \\
\hline Quinonas & - \\
\hline Taninos & + \\
\hline Cardiotónicos & + \\
\hline Saponinas & - \\
\hline + = Detectado (prueba positiva) - = No detectado
\end{tabular}

La identificación de terpenos arrojó resultados positivos. Se realizó la caracterización cualitativa haciendo uso de la prueba de Lieberman Burchard y utilizando como patrón colesterol. De esta manera 

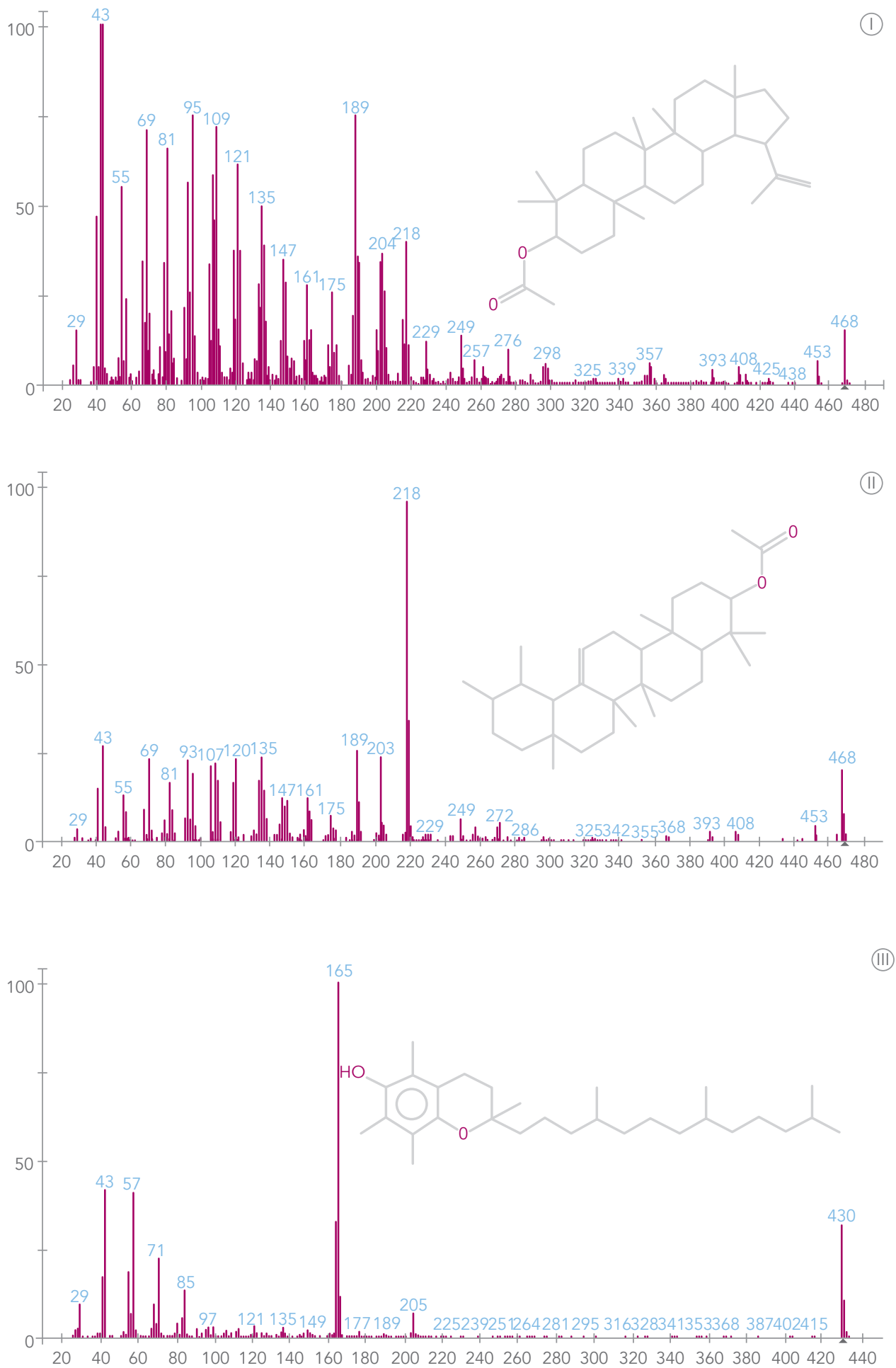


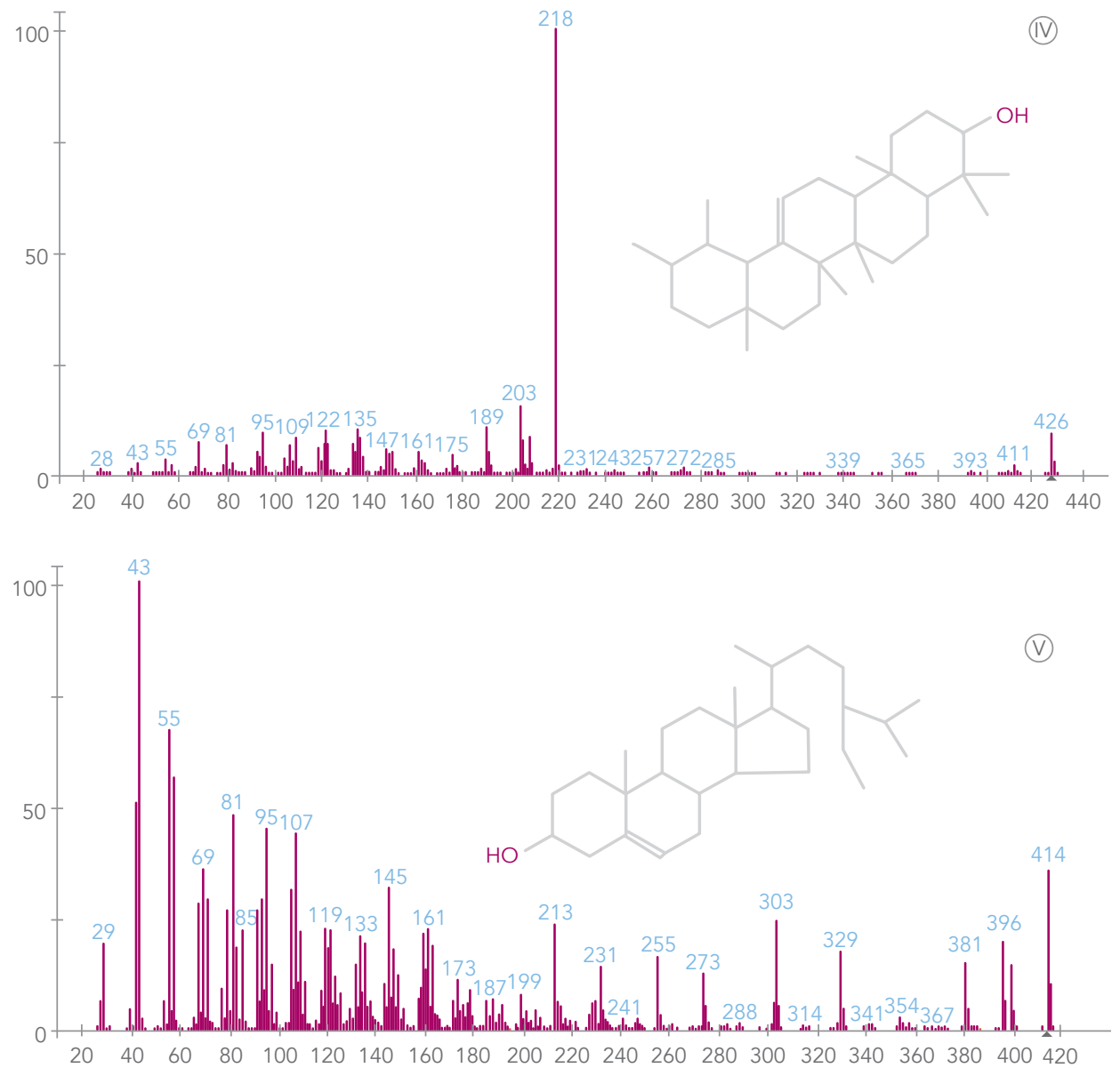

Figura 1. A) Estructuras de los compuestos aislados de la extracto soluble en EdP (1-3) y del extracto etanólico (4-5); B) Espectros de masas a 70 eV de los compuestos aislados 1-5.

se observó que cuando se trata tanto el patrón como el extracto de EdP con el reactivo mencionado se observa una coloración que va desde azul hasta verde intenso dando positiva esta prueba para terpenos y esteroles (Shreya-Mandal et al., 2013).

Los resultados preliminares están acorde con los resultados reportados para Salvia apiana (Luis et al., 1996), en donde se encontraron terpenoides apiánanos (Saficinólido, Salvigenina) y una nueva sustancia, cuya estructura fue establecida por métodos espectroscópicos, resultando asimismo ser el primer ejemplo de otro nuevo tipo de esqueleto carbonado C23-terpenico cuya serie se ha denominado Hassananos (Luis et al., 1996) Otras especies como Salvia amarissima (Esmeralda et al., 2010) han reportado grupos de metabolitos del tipo carotenoide, flavonoide y cumarina. Finalmente el estudio de Salvia uruapana (Manjarréz et al., 2003) muestra fracciones ricas en glucósidos y un compuesto sesquiterpenico denominado tonalesina, el cual ya había sido reportado en Salvia tonalensis (Manjarréz et al., 2003). 

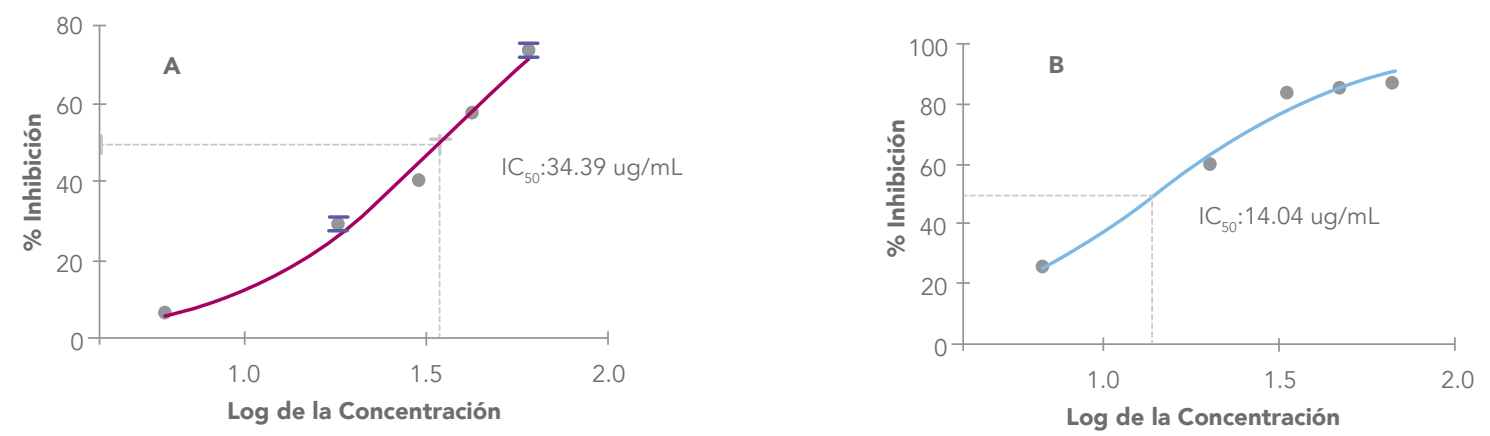

Figura 2. Curvas dosis-respuesta para A) compuesto 3; B) ácido ascórbico

\section{Fraccionamiento y Purificación}

Extracto soluble en éter de petróleo

Una vez detectados los grupos de metabolitos, se procedió al fraccionamiento del extracto soluble en EdP. Para ello se realizó una CC en fase normal sobre gel de sílice, el monitoreo se llevó a cabo por CCD. Después de purificaciones sucesivas, se obtuvieron tres compuestos de tipo terpenoide, los cuales se analizaron por CG-EM y respectiva comparación con la librería NIST-08, la cual arrojó porcentajes de coincidencia mayores al $95 \%$, cuyas estructuras y espectros de masas se exponen en la Figura 1.

Compuesto 1 (F.1.2.1): Sólido blanco (13,3 mg), obtenido mediante purificación por CC, eluido con Hex: $\mathrm{Me}_{2} \mathrm{CO}$ (7:3) (Rf en CCD equivalente a 0,74). El compuesto dió prueba positiva al reactivo de Lieberman-Burchard y al reactivo en placa con vainillina$\mathrm{H}_{2} \mathrm{SO}_{4}$, lo que indica que posiblemente se trata un compuesto con núcleo triterpénico.

El análisis del espectro de masas permite establecer la fórmula molecular $\mathrm{C}_{32} \mathrm{H}_{52} \mathrm{O}_{2}$, a partir del ion

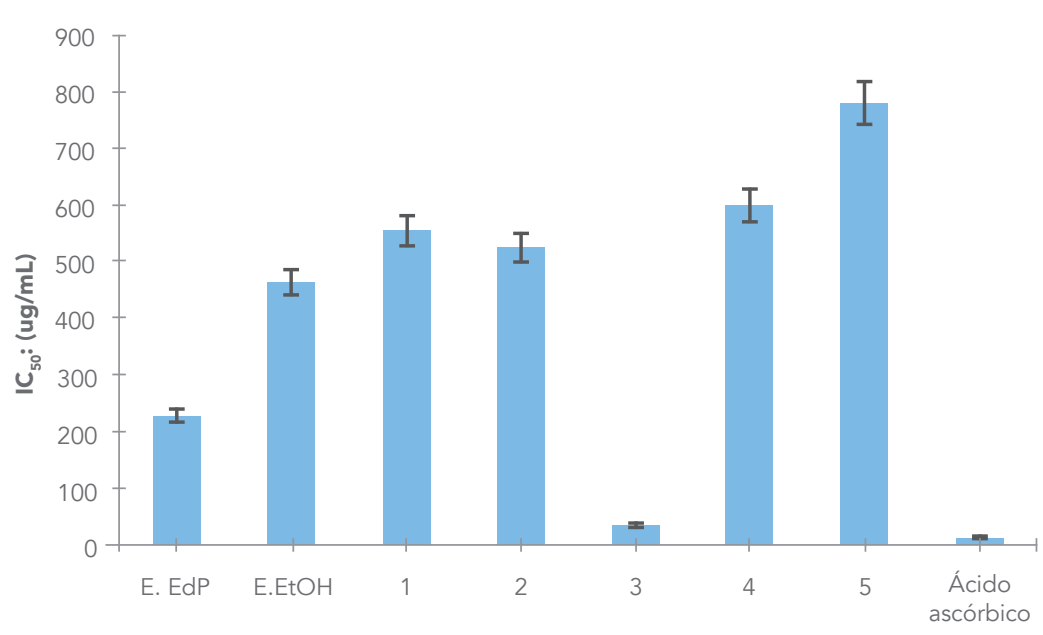

Figura 3. Concentración inhibitoria media de los extractos crudos y los compuestos aislados. 
molecular m/z 468. Los fragmentos más importantes para el diagnóstico estructural son: $\mathrm{m} / \mathrm{z} 249$, que coincide con la eliminación de parte de la estructura hidrocarbonada correspondiente a los anillos C (parcialmente), D, E y el grupo isoprenilo. El fragmento m/z 189 (249-AcOH), que corresponde a la eliminación del grupo acetato. El patrón de fragmentación del compuesto 1 pertenece a las rupturas típicas de triterpenos pentacíclicos (César et al., 2009), que de acuerdo con lo mencionado, este compuesto fue identificado como con el acetato de lupeol (1), cuya comparación con la librería NIST-08 mostró un 97\% de coincidencia.

Compuesto 2 (F.1.2.4): Sólido marrón (5,6 mg), obtenido por CC utilizando un sistema cromatografico Hex-Me ${ }_{2} \mathrm{CO}$ (7:3) como fase móvil (Rf en CCD de 0,80$)$. Igualmente se sometió a las pruebas de Lieberman-Burchard y revelado en placa con VainiIlina- $\mathrm{H}_{2} \mathrm{SO}_{4}$, cuyo resultado en ambos casos fue positivo, lo cual es característico para compuestos del tipo triterpeno.

El Espectro de masas permitió establecer el patrón de fragmentación en la cual se observó la presencia del ion molecular $\mathrm{m} / \mathrm{z} 468$, con la fórmula molecular, $\mathrm{C}_{32} \mathrm{H}_{52} \mathrm{O}_{2}$. Los fragmentos $\mathrm{m} / \mathrm{z} 249,218$, 203, y 189 constituyeron a los denominados picos diagnóstico para esqueletos tipo amirina. El fragmento m/z 218 es el más representativo, porque corresponde a un reordenamiento Retro-Diels-Alder (RDA), en el anillo que posee el doble enlace, siendo una evidencia de la existencia de esa instauración. De acuerdo al anterior análisis, este compuesto fue identificado como el acetato de a-amirina (2), cuya comparación con la librería NIST-08 mostró un 95\% de coincidencia.

Compuesto 3 (F.1.3.1): Sólido blanco (7,1 mg), soluble en $\mathrm{EdP}$ y $\mathrm{CHCl}_{3}$, obtenido por CC utilizando como fase móvil Hex:Me ${ }_{2} \mathrm{CO}$ (7:3). El espectro de masas muestra una señal a 430 uma, lo que es indicativo de la señal correspondiente al ion molecular. Se observa el pico base con m/z 165 formado por la ruptura del anillo saturado contiguo al anillo de benceno sustituido. Se presentó el ion de m/z 205 relacionado con la pérdida de la cadena lateral hidrocarbonada. El análisis por espectrometría de masas (EM) a 70 eV y la respectiva comparación con la biblioteca NIST-08 reporto un 96\% de concordancia con a-tocoferol (3).

\section{Extracto soluble en Etanol}

Las fracciones obtenidas del extracto $\mathrm{EtOH}$ por fraccionamiento liquido-liquido (solubles en EdP, diclorometano (DCM), acetato de etilo (AcOEt) y un residuo hidroalcohólico) fueron monitoreadas por CCD. La fracción soluble en DCM resultó ser más promisoria dado que mostró un mejor perfil en

\section{El Espectro de masas permitió establecer el patrón de} fragmentación en la cual se observó la presencia del ion molecular $\mathrm{m} / \mathrm{z}$ 468, con la formula molecular, $\mathrm{C}_{32} \mathrm{H}_{52} \mathrm{O}_{2}$. Los fragmentos $\mathrm{m} / \mathrm{z} 249,218,203$, y 189 constituyeron a los denominados picos diagnóstico para esqueletos tipo amirina. 
compuestos de interés (triterpenos y esteroides), a razón de la prueba de Lieberman-Burchard y al reactivo en placa con vainillina- $\mathrm{H}_{2} \mathrm{SO}_{4}$. A continuación se muestran los compuestos mayoritarios de la fracción soluble en DCM, separados por cromatografía en columna empleando como fase móvil DCM-AcOEt (7:3) e identificados por EM.

Compuesto 4 (F.1.4.1): Corresponde a un sólido blanco que peso $6.3 \mathrm{mg}$, soluble en $\mathrm{EdP}$ y $\mathrm{CHCl}_{3^{\prime}}$ eluido tras una CC cuya fase móvil fue DCM-AcOEt $(7: 3)(R f=0.64)$. Este compuesto fue revelado con Vainillina- $\mathrm{H}_{2} \mathrm{SO}_{4}$ y Lieberman-Burchard, dando prueba positiva para ambos casos, indicando que probablemente se trata de un triterpeno.

El espectro de masas mostró el ion molecular en $\mathrm{m} / \mathrm{z} 426$ acorde a la fórmula molecular $\mathrm{C}_{30} \mathrm{H}_{50} \mathrm{O}$. Se observa un pico de pequeña intensidad con $\mathrm{m} / \mathrm{z}$ 411, correspondiente a la pérdida de un grupo $\mathrm{CH}_{3}$ a partir del ion molecular, siendo el pico base del espectro m/z 218, el cual se forma tras una reacción de Retro Diels-Alder en el anillo C, a partir del cual se originan los iones con m/z 203 por la pérdida de un grupo $\mathrm{CH}_{3}$ y m/z 189, debido a la pérdida del grupo $\mathrm{C}_{2} \mathrm{H}_{5}$ por la ruptura del enlace que une a los anillos D y E. También se presenta un ion a m/z 207 que contiene a los anillos A y $\mathrm{B}$. Los resultados obtenidos están en correspondencia con lo reportado por (César et al., 2009) para la a-amirina, cuya comparación con la librería NIST-08 arrojo un 95\% de coincidencia.

Compuesto 5 (F. 8.1.1): Corresponde a un sólido gris $(4,1 \mathrm{mg})$ fue monitoreada por CCD utilizando una mezcla de disolventes DCM-MeOH 8:2 (Rf = $0,68)$ y posteriormente revelada con Vainillina- $\mathrm{H}_{2} \mathrm{SO}_{4^{\prime}}$ generando un punto de color morado, indicando la presencia de terpenos, para lo cual se confirmó con la prueba de Lieberman-Burchard.

En el espectro de masas muestra el perfil de fragmentación característica de esteroides, en el cual se observaron señales a m/z 396, correspondientes a un fragmento del esteroide con una pérdida de una molécula de agua ( $\left.\mathrm{m} / \mathrm{z} \mathrm{M}^{+}-18\right)$, (proveniente del grupo $\mathrm{OH}$ de la posición 3). Este fragmento sufre una nueva fragmentación dando origen al fragmento a m/z 255, el cual se forma por la pérdida de la cadena lateral unida carbono 17. Por otra parte, se ven claramente el fragmento del esteroide a $273 \mathrm{~m} / \mathrm{z}$ obtenido a partir de la pérdida de la cadena lateral por parte del esteroide original y la posterior perdida de una molécula de agua ( $\left.\mathrm{m} / \mathrm{z} \mathrm{M}^{+}-18\right)$, proveniente del grupo $\mathrm{OH}$ de la posición 3. De esta manera el compuesto 5 fue identificado como el y-sitosterol, cuya comparación con la librería NIST-08 arrojo un 95\% de coincidencia.

A nivel Colombia, es la primera vez que se realizan trabajos con la especie vegetal Salvia leucantha, con lo cual se está contribuyendo de forma preliminar a la quimio- taxonomía del género y de la familia; los compuestos identificados por CG-EM son muy comunes en la mayoría de especies vegetales como en las hojas de Pentacalia vaccinioides y en las hojas de propóleos. 
Evaluación de la Capacidad Antioxidante

Se determinó la capacidad antioxidante de los extractos crudos y fracciones obtenidas. Esta evaluación se desarrolló mediante la determinación del $I_{50}$ a través de las curvas dosis-respuesta (Figura 2) generadas por el método de DPPH, cuyos resultados se muestran en la Figura 3. Los datos obtenidos fueron comparados frente a un patrón (ácido ascórbico), cuya concentración inhibitoria media fue $14.04 \mu \mathrm{g} / \mathrm{mL}$ (0,080 M). Así mismo, el extracto etanólico y sus fracciones derivadas exhibieron valores bajos de capacidad antioxidante. Sin embargo, al analizar el extracto soluble EdP se encontraron valores de $\mathrm{IC}_{50}$ más altos. El compuesto 3 mostró un valor molar de $\mathrm{IC}_{50}$ equivalente al del patrón $(0,08 \mathrm{M})$, lo cual concuerda con lo reportado ya que se conoce que el a-tocoferol, un derivado de la vitamina $E$, se encuentra catalogado como un compuesto antioxidante.

\section{DISCUSIÓN}

A nivel Colombia, es la primera vez que se realizan trabajos con la especie vegetal Salvia leucantha, con lo cual se está contribuyendo de forma preliminar a la quimio- taxonomía del género y de la familia; los compuestos identificados por CG-EM son muy comunes en la mayoría de especies vegetales como en las hojas de Pentacalia vaccinioides y en las hojas de propóleos (César et al., 2009). Dada la abundancia reconocida de los triterpenos de origen vegetal, se han reportado diversas propiedades de estos compuestos dependientes de la dosis, entre las que se resaltan el efecto anticancerígeno (Reyes, 2007), función hepática y biliar (López-Carreras et al., 2012) y propiedades antihipertensivas y antiinflamatorias (Bonkanka-Tabares, 2009).

El estudio fitoquímico preliminar realizado a la especie Salvia leucantha dió como resultado la identificación de triterpenoides como acetato de lupeol, $\boldsymbol{\alpha}$-amirina, acetato de $\boldsymbol{\alpha}$-amirina y $\boldsymbol{\gamma}$-sitosterol, indicando que esta especie, al igual que otras del mismo género y familia, son ricas en triterpenos como Salvia argéntea (Lakhal et al., 2014), Salvia chinensis (Wang et al., 2009), Salvia multicaulis (Ulubelen et al, 1998), Salvia viridis (Rungsimakan \& Rowan, 2014), Salvia glutinosa (Topcu et al., 1997) y Salvia przewalskii (Xue et al., 2014).

Finalmente, se observa que la especie vegetal Salvia leucantha presenta niveles relativamente bajos de capacidad antioxidante, de acuerdo con los resultados obtenidos mediante evaluación por el método de captación de radicales DPPH. Algunas especies del genero Salvia han mostrado actividad antioxidante, tales como Salvia officinalis y Salvia fruticosa (Kontogianni et al., 2013). Sin embargo, la actividad de estas dos plantas fue correlacionada con el contenido de fenoles presentes en los extractos (Kruma, Andjelkovic, Verhe, \& Kreicbergs, 2008), mientras que en el caso de Salvia leucantha el contenido de derivados de la vitamina E, más específicamente el compuesto 3 ( $\boldsymbol{\alpha}$-tocoferol) fue quien mostró bioactividad y dió buenos resultados al ser comparados con el ácido ascórbico; Estos resultados están acorde con lo reportado en la literatura para todos los derivados de la vitamina $\mathrm{E}$ (Thoo et al., 2013).

\section{AGRADECIMIENTOS}

De forma especial se agradece a la Universidad Distrital Francisco José de Caldas por la financiación del Proyecto de investigación, así como al Grupo de Investigación en Productos Naturales Vegetales en cuyo Laboratorio se desarrolló todo el proceso experimental. 


\section{REFERENCIAS}

1. Fernández-Alonso, J.L. 2006. Revisión taxonómica de Salvia sect. Siphonantha (Labiatae). Anales del Jardín Botánico de Madrid, 63:145-157.

2. Bonkanka-Tabares C. X. 2009. Evolución farmacológica de terpenos y flavonoides de origen vegetal, ISBN: 978-84-7756-779-0, 1, 24-28.

3. Brand-Williams W., Cuvelier M. E., Berset C. 1995. Use of a free radical method to evaluate antioxidant activity. LWT - Food Science and Technology, 28:25-30.

4. López-Carreras N., Miguel M., Aleixandre A. 2012. Propiedades beneficiosas de los terpenos iridoides sobre la salud. Nutrición Clínica y Dietética Hospitalaria, 32:81-91.

5. César J., Pérez B., Best C. R., Llanes F. 2009. Triterpenos pentacíclicos en el propóleo. Revista de la Sociedad Química del Perú, 75:439-452.

6. Esmeralda C., Ferrer L., Guadalupe M., Dirzo S., Baez D. A., Humberto J., García R. 2010. Estudio preliminar fitoquímico y de la actividad antimicrobiana de Salvia amarissima Ort. Investigación Universitaria Multidisciplinaria: Revista de Investigación de la Universidad Simón Bolívar, 9:67-76.
7. Kontogianni V.G., Tomic G., Nikolic I., Nerantzaki A.A., Sayyad N., Stosic-Grujicic S., Stojanovic I., Gerothanassis I.P., Tzakos A.G. 2013. Phytochemical profile of Rosmarinus officinalis and Salvia officinalis extracts and correlation to their antioxidant and anti-proliferative activity. Food Chemistry, 136:120-9.

8. Kruma Z., Andjelkovic M., Verhe R., Kreicbergs V. 2008. Phenolic compounds in oils aromatised with basil, oregano and thyme. Proceedings of International Conference of Agricultural Engineering, 1:99-103.

9. Lakhal H., Kabouche A., Alabdul Magid A. Voutquenne-Nazabadioko L., Harakat D., Kabouche Z. 2014. Triterpenoids from Salvia argentea var. aurasiaca (Pomel) Batt. \& Trab. and their chemotaxonomic significance. Phytochemistry, 102:145-151.

10. Luis J. G., Lahlou E. H., Andres L. S. 1996. Hassananes: C23 terpenoids with a new type of skeleton from Salvia apiana Jeps. Tetrahedron, 52:12309-12312.

11. Manjarréz R., Uribe B. A. F., Cárdenas J., Salvia D., Frontana-uribe B. A. 2003. Estudio fitoquímico de Salvia uruapana. Journal of the Mexican Chemical Society, 47:207-209. 
12. Reyes F. J. 2007. Caracterizacion del efecto anticancerígeno del ácido maslínico, triterpeno pentacíclico de origen natural. Universidad de Granada, Tesis Doctoral, pp 157-170.

13. Rungsimakan S., Rowan M. G. 2014. Terpenoids, flavonoids and caffeic acid derivatives from Salvia viridis L. cvar. Blue Jeans. Phytochemistry. doi:10.1016/j. phytochem.2014.08.029

14. Mandal S., Patra A., Samanta A., Roy S., Mandal A., Mahapatra T.D., Pradhan S., Das K., Nandi D.K. 2013. Analysis of phytochemical profile of Terminalia arjuna bark extract with antioxidative and antimicrobial properties. Asian Pacific Journal of Tropical Biomedicine, 3:960-966.

15. Thoo Y.Y., Abas F., Lai O.M., Ho C.W., Yin J., Hedegaard R.V., Skibsted L.H., Tan C.P.. 2013. Antioxidant synergism between ethanolic Centella asiatica extracts and $\boldsymbol{\alpha}$-tocopherol in model systems. Food Chemistry, 138:1215-1219.

16. Topcu G., Tan N., Kökdil G., Ulubelen A. 1997. Terpenoids from Salvia glutinosa. Phytochemistry, 45:1293-1294.
17. Ugochukwu S. C., Uche A., Ifeanyi, O. 2013. Preliminary phytochemical screening of different solvent extracts of stem bark and roots of Dennetia tripetala G. Baker. Asian Journal of Plant Science and Research, 3:10-13.

18. Ulubelen A., Nur T., Sonmez U., Topcu G. 1998. Diterpenoids and triterpenoids from Salvia multicaulis. Phytochemistry, 47:899-901.

19. Wang Y., Song D., Li Z., Yuan T., Zhang H., Pei Y., Ying Y., Hua H. 2009. Triterpenoids isolated from the aerial parts of Salvia chinensis. Phytochemistry Letters, 2:81-84.

20. Xue Y., Wu Y., Zhu H., Li X.N., Qian J.F., Lai Y., Chen C., Yao G., Luo Z., Li Y., Zhang Y. 2014. Salviprzols A and B, C21- and C22-terpenoids from the roots of Salvia przewalskii Maxim. Fitoterapia, 99:204-210. 